\title{
Algebraic Properties of Implication-Based Anti-Fuzzy Subgroup over a Finite Group
}

\author{
M. Selvarathi
}

\begin{abstract}
This article deals with few algebraic characteristics of implication-based anti-fuzzy subgroup of a finite group.In addition, the implication-based anti-fuzzy direct product of implication-based anti-fuzzy subgroups over finite groups is developed and studied elaborately. The condition for an implication-based anti-fuzzy subgroup of a finite group to be a conjugate to another implication-based anti-fuzzy subgroup is conceptualized. Some of their characteristics are investigated in this paper.
\end{abstract}

Keywords: Implication-based anti-fuzzy subgroup, Implication-based anti-fuzzy normal subgroup, Implication-based anti-fuzzy direct product.

\section{INTRODUCTION}

\section{Zadeh[16] first introduced the concept of fuzzy set in} 1965. In 1971, Rosenfeld [10] applied the theory of fuzzy sets developed by Zadeh to groups and framed the elementary theory of groupoids and groups. Henceforth, intensive research was elaborately done in this field. The algebraic structures of fuzzy subgroups were studied by [7], [3], [4] and many other researchers. In 1990, Biswas [1] postulated the theory of anti-fuzzy subgroups of groups [8], [5], [15], [9], [2], [13] and many others studied anti-fuzzy subgroups in detail. In 2015, M. Selvarathi [11], [12] studied about implication-based fuzzy normal subgroup and implication-based anti-fuzzy subgroup over a finite group. In this paper, further research had been done on implication-based anti-fuzzy subgroups and their direct product. Few basic properties of them are also proved.

\section{PRELIMINARIES}

\section{Definition II.1[10]}

$\operatorname{Let}(\Omega, \cdot)$ be a group. Let a fuzzy set in $\Omega$ be a function A from $\Omega$ to $[0,1]$. A will be known as fuzzy subgroup of $\Omega$ if for all $\xi_{1}, \xi_{2}$ in $\Omega$

(i) $A\left(\xi_{1} \xi_{2}\right) \geq \min \left(A\left(\xi_{1}\right), A\left(\xi_{2}\right)\right)$

(ii) $A\left(\xi_{1}^{-1}\right) \geq A\left(\xi_{1}\right)$

Revised Manuscript Received on December 22, 2019

* Correspondence Author

M. Selvarathi*, Department of Mathematics, Karunya Institute of Technology and Sciences (Deemed to be University), Coimbatore, India. Email: selvarathi.maths@gmail.com
Let $\chi$ be an universe of discourse and $(\Omega, \cdot)$ be a group. In fuzzy

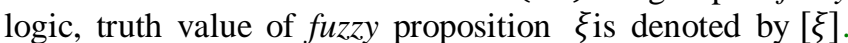
The fuzzy logical and the corresponding set theoretical notations used in this paper are

$$
\begin{array}{ll}
\text { (i) } & (\xi \in A)=A(\xi) ; \\
\text { (ii) } & (\psi \wedge \tau)=\min \{[\psi],[\tau]\} ; \\
\text { (iii) } & (\psi \rightarrow \tau)=\min \{1,1-[\psi]+[\tau]\} ; \\
\text { (iv) } & (\forall \xi \psi(\xi))=\inf _{\xi \in \chi}[\psi(\xi)] ; \\
\text { (v) } & (\exists \xi \psi(\xi))=\sup _{\xi \in \chi}[\psi(\xi)] ;
\end{array}
$$

\section{Definition II.2[14]}

If a fuzzy subset $A$ of a group $\Omega$ satisfies for any $\xi_{1}, \xi_{2} \in \Omega$

$$
\text { (i) } \vDash\left(\xi_{1} \in A\right) \wedge\left(\xi_{2} \in A\right) \rightarrow\left(\xi_{1} \xi_{2} \in A\right)
$$$$
\text { (ii) } \vDash\left(\xi_{1} \in A\right) \rightarrow\left(\xi_{1}{ }^{-1} \in A\right)
$$

Then $A$ is called a fuzzifying subgroup.

The concept of $\lambda$ - tautology is $\vDash_{\lambda} \psi$ if and only if $(\psi) \geq \lambda$ for all valuation by Ying [6]

\section{Definition II.3[14]}

Let $A$ be a fuzzy subset of a finite group $\Omega$ and $\lambda \in(0,1]$ is a fixed number. If for any $\xi_{1}, \xi_{2} \in \Omega$

$$
\begin{array}{ll}
\text { (i) } & \vDash_{\lambda}\left(\xi_{1} \in A\right) \wedge\left(\xi_{2} \in A\right) \rightarrow\left(\xi_{1} \xi_{2} \in A\right) \\
\text { (ii) } & \vDash_{\lambda}\left(\xi_{1} \in A\right) \rightarrow\left(\xi_{1}{ }^{-1} \in A\right)
\end{array}
$$

Then $A$ is called an implication-based fuzzy subgroup of $\Omega$.

\section{Definition II.4[1]}

Let $\Omega$ be a group. A fuzzy subset $\mu$ of $\Omega$ is called an anti-fuzzy subgroup of $\Omega$ if for $\xi, \psi \in \Omega$

(i) $\mu(\xi \psi) \leq \mu(\xi) \mu(\psi)$

(ii) $\mu\left(\xi^{-1}\right) \leq \mu(\xi)$

\section{Definition II.5 [12]}

Let $A$ be a fuzzy subset of a finite group $\Omega$ and $\lambda \in(0,1]$ is a fixed number. If for any $\xi, \psi \in \Omega$

$\vDash_{\lambda}(\xi \psi \in A) \rightarrow((\xi \in A) \vee(\psi \in A))$

$\vDash_{\lambda}\left(\xi^{-1} \in A\right) \rightarrow(\xi \in A)$

Then $A$ is called an implication-based anti-fuzzy subgroup of $\Omega$.

\section{Example II.1 [12]}

Consider the finite group $\Omega=\{\varepsilon, \alpha, \beta, \gamma\}$ along with the binary operation ' $*$ ' whose closure table is as follows. 


\begin{tabular}{|c|c|c|c|c|}
\hline$*$ & $\varepsilon$ & $\alpha$ & $\beta$ & $\gamma$ \\
\hline$\varepsilon$ & $\varepsilon$ & $\alpha$ & $\beta$ & $\gamma$ \\
\hline$\alpha$ & $\alpha$ & $\varepsilon$ & $\gamma$ & $\beta$ \\
\hline$\beta$ & $\beta$ & $\gamma$ & $\varepsilon$ & $\alpha$ \\
\hline$\gamma$ & $\gamma$ & $\beta$ & $\alpha$ & $\varepsilon$ \\
\hline
\end{tabular}

For the fuzzy set $A: \Omega \rightarrow[0,1]$ defined by $A(\varepsilon)=0.2, A(\alpha)=0.3, A(\beta)=0.4, A(\gamma)=0.5 \quad$ with $\lambda=$.4and the implication operator is that of Kleene-Dienes, we have

\begin{tabular}{|c|c|c|c|c|}
\hline$\vee$ & {$[\varepsilon]$} & {$[\alpha]$} & {$[\beta]$} & {$[\gamma]$} \\
\hline$[\varepsilon]$ & 0.2 & 0.3 & 0.4 & 0.5 \\
\hline$[\alpha]$ & 0.3 & 0.3 & 0.4 & 0.5 \\
\hline$[\beta]$ & 0.4 & 0.4 & 0.4 & 0.5 \\
\hline$[\gamma]$ & 0.5 & 0.5 & 0.5 & 0.5 \\
\hline
\end{tabular}

Then $A$ is an implication-based anti-fuzzy subgroup of $\Omega$.

\section{Definition II.6 [12]}

Let $A$ is an implication-based anti-fuzzy subgroup of a group $\Omega . A$ is called an implication-based anti-fuzzy normal subgroup of a group $\Omega$ if $\vDash_{\lambda}(\xi \psi \in A) \rightarrow(\psi \xi \in A)$ for all $\xi, \psi \in \Omega$

\section{Definition II.7 [12]}

Let $A$ and $B$ be two implication-based anti-fuzzy subgroups of the finite group $\Omega$, then the internal product $A \cdot B$ is defined by

$$
\begin{gathered}
\vDash_{\lambda}(\forall \psi, \tau\{(\psi \in A) \vee(\tau \in B)\} ; \psi \tau=\xi ; \psi, \tau \in \Omega) \\
\rightarrow(\xi \in A \cdot B), \quad \xi \in \Omega
\end{gathered}
$$

Theorem II.1 [12]

$A$ is an implication-based fuzzy subgroup of the group $\Omega$ if and only if its complement $A^{C}$ is an implication-based anti-fuzzy subgroup of $\Omega$.

\section{Theorem II.2 [12]}

Intersection of two implication-based anti-fuzzy subgroups of a group $\Omega$ is again an implication-based anti-fuzzy subgroup of $\Omega$.

\section{Theorem II.3 [12]}

Intersection of two implication-based fuzzy subgroups of a group $\Omega$ is an implication-based fuzzy subgroup of $\Omega$.

\section{Theorem II.4 [12]}

$A$ is an implication-based anti-fuzzy subgroup of $\Omega$ if and only iffor all $\xi, \psi \in \Omega$

$$
\vDash_{\lambda}\left(\xi \psi^{-1} \in A\right) \rightarrow((\xi \in A) \vee(\psi \in A))
$$

Theorem II.5 [12]

If $A$ is an implication-based anti-fuzzy subgroup of $\Omega$ with the identity element ' $\varepsilon$ 'then for all $\xi \in \Omega$

$$
\begin{array}{ll}
\text { (i) } & \vDash_{\lambda}(\varepsilon \in A) \rightarrow(\xi \in A) \\
\text { (ii) } & \vDash_{\lambda}(\xi \in A) \rightarrow\left(\xi^{-1} \in A\right)
\end{array}
$$

Theorem II.6 [12]
If $B$ is an implication-based anti-fuzzy normal subgroup of the finite group $\Omega$ and $C$ is an implication-based anti-fuzzy subgroups of $\Omega$ then

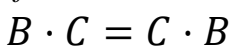

(ii)

$$
\text { subgroup of } \Omega
$$

Hereafter let $(\Omega, \cdot)$ denote a group and ' $\varepsilon$ ' be its identity element. And let $\lambda \in(0,1]$ be a fixed number.

\section{RESULT ANALYSIS OF IMPLICATION-BASED ANTI-FUZZY SUBGROUPS OVER A FINITE GROUP}

\section{Theorem III.1}

Let $A$ and $B$ be implication-based anti-fuzzy subgroups of a finite group $\Omega$. Then $A \cup B$ is also an implication-based anti-fuzzy subgroup of a finite group.

Proof:

Let $A$ and $B$ be implication-based anti-fuzzy subgroups of a finite group $\Omega$.

By Theorem II. $1, A^{C}$ and $B^{C}$ are implication-based fuzzy subgroups of a finite group $\Omega$.

By Theorem II.3, $A^{C} \cap B^{C}$ are implication-based fuzzy subgroups of a finite group $\Omega$.

By De Morgan's Law

$$
\begin{gathered}
\vDash_{\lambda}\left(\xi \in A^{C} \cap B^{C}\right) \wedge\left(\psi \in A^{C} \cap B^{C}\right) \\
\rightarrow\left(\xi \psi^{-1} \in A^{C} \cap B^{C}\right)
\end{gathered}
$$

$$
\begin{gathered}
\vDash_{\lambda}\left(\xi \in(A \cup B)^{C}\right) \wedge\left(\psi \in(A \cup B)^{C}\right) \\
\rightarrow\left(\xi \psi^{-1} \in(A \cup B)^{C}\right) \\
\vDash_{\lambda}(1-(\xi \in(A \cup B))) \wedge(1-(\psi \in(A \cup B))) \\
\rightarrow\left(1-\left(\xi \psi^{-1} \in(A \cup B)\right)\right) \\
\left.\vDash_{\lambda}\left(\xi \psi^{-1} \in(A \cup B)\right)\right) \\
\rightarrow 1-((1-(\xi \in(A \cup B))) \wedge(1-(\psi \in(A \cup B)))) \\
\left.\vDash_{\lambda}\left(\xi \psi^{-1} \in(A \cup B)\right)\right) \\
\rightarrow(\xi \in(A \cup B)) \vee(\psi \in(A \cup B))
\end{gathered}
$$

By Theorem II.4, Therefore $A \cup B$ is an implication-based anti-fuzzy subgroup of a finite group $\Omega$.

\section{Definition III.1}

Let $A$ be an implication-based anti-fuzzy subgroup of a finite group $\Omega$ and $\varphi: \Omega \rightarrow \Omega$ be a function defined on $\Omega$. Then the implication-based anti-fuzzy subgroup $B$ of $\varphi(\Omega)$ is defined by $\vDash_{\lambda}\left(\forall \xi\{(\xi \in A)\} ; \xi=\varphi^{-1}(\psi)\right) \rightarrow(\psi \in B)$ for all $\psi \in \varphi(\Omega)$.

Similarly, if $B$ is an implication-based anti-fuzzy subgroup of $\varphi(\Omega)$ then the implication-based anti-fuzzy subgroup $A=\varphi \circ B$ in $\Omega$ is defined as $\vDash_{\lambda}(\varphi(\xi) \in B) \rightarrow(\xi \in A)$ for all $\xi \in \Omega$, and is called the pre-image of $B$ under $\varphi$.

\section{Definition III.2}

An implication-based anti-fuzzy subgroup $A$ of a finite group $\Omega$ is said to have infremum - property if for any $B \subseteq \Omega$,there exists $\eta_{0} \in B$ such

that $_{\lambda}(\forall \eta\{(\eta \in A)\} ; \eta \in B) \rightarrow\left(\eta_{0} \in A\right)$.

\section{Theorem III.2}

Let $A$ be an implication-based anti-fuzzy subgroup of a finite group $\Omega$. Then $A$ is an implication-based anti-fuzzy normal subgroup of $\Omega$ if and only if $\vDash_{\lambda}\left(\xi^{-1} \psi^{-1} \xi \psi \in A\right) \rightarrow(\xi \in A)$ for all $\xi, \psi \in \Omega$.

Proof: 
Let $\xi, \psi \in \Omega$.

(i) $\Rightarrow$ (ii)

$A$ is an implication-based fuzzy normal subgroup of the group

$\Omega$.

$\vDash_{\lambda}\left(\xi^{-1} \psi^{-1} \xi \psi \in A\right)$

$$
\begin{gathered}
\rightarrow\left(\psi^{-1} \xi \psi \cdot \xi^{-1} \in A\right) \\
\rightarrow\left(\psi^{-1} \xi \psi \in A\right) \vee\left(\xi^{-1} \in A\right) \\
\rightarrow(\xi \in A) \vee(\xi \in A) \\
\rightarrow(\xi \in A)
\end{gathered}
$$

Conversely $(i i) \Rightarrow(i)$

Assume that

$\vDash_{\lambda}\left(\xi^{-1} \psi^{-1} \xi \psi \in A\right) \rightarrow(\xi \in A)$ for all $\xi, \psi \in \Omega$.

Let $\xi, \psi \in \Omega$.

$\vDash_{\lambda}\left(\xi^{-1} \psi \xi \in A\right)$

$$
\begin{gathered}
\rightarrow\left(\psi \psi^{-1} \cdot \xi^{-1} \psi \xi \in A\right) \\
\rightarrow\left(\psi \cdot \psi^{-1} \xi^{-1} \psi \xi \in A\right) \\
\rightarrow(\psi \in A) \vee\left(\psi^{-1} \xi^{-1} \psi \xi \in A\right) \\
\rightarrow(\psi \in A)
\end{gathered}
$$

Also

$\vDash_{\lambda}(\psi \in A)$

$$
\begin{aligned}
& \rightarrow\left(\xi \xi^{-1} \cdot \psi \cdot \xi \xi^{-1} \in A\right) \\
& \rightarrow\left(\xi \cdot \xi^{-1} \psi \xi \cdot \xi^{-1} \in A\right) \\
& \rightarrow(\xi \in A) \vee\left(\xi^{-1} \psi \xi \in A\right) \vee\left(\xi^{-1} \in A\right) \\
& \rightarrow(\xi \in A) \vee\left(\xi^{-1} \psi \xi \in A\right)
\end{aligned}
$$

Therefore $\vDash_{\lambda}(\psi \in A) \rightarrow(\xi \in A) \vee\left(\xi^{-1} \psi \xi \in A\right)$

Case (i): Suppose

$\vDash_{\lambda}(\xi \in A) \rightarrow(\xi \in A) \vee\left(\xi^{-1} \psi \xi \in A\right)$

$\Rightarrow k_{\lambda}(\xi \in A) \rightarrow(\psi \in A)$ for all $\xi, \psi \in \Omega$

$\Rightarrow \vDash_{\lambda}(\xi \psi \in A) \rightarrow(\psi \xi \in A)$ for all $\xi, \psi \in \Omega$

Case(ii): Suppose

$\vDash_{\lambda}\left(\xi^{-1} \psi \xi \in A\right) \rightarrow(\xi \in A) \vee\left(\xi^{-1} \psi \xi \in A\right)$

$\Rightarrow \vDash_{\lambda}\left(\xi^{-1} \psi \xi \in A\right) \rightarrow(\psi \in A)$ for all $\xi, \psi \in \Omega$

$\Rightarrow \vDash_{\lambda}(\xi \psi \in A) \rightarrow(\psi \xi \in A)$ for all $\xi, \psi \in \Omega$

Therefore, $A$ is an implication-based anti-fuzzy normal subgroup of the group $\Omega$.

\section{Theorem III.3}

A homomorphic image or pre-image of an implication-based anti-fuzzy subgroup of a finite group $\Omega$ is also an implication-based anti-fuzzy subgroup provided in the former case the infremum property holds.

Proof:

Let $A$ be an implication-based anti-fuzzy subgroup of a finite group $\Omega$ for which the infremum property holds. Let $f: G \rightarrow G$ be a homomorphism.

For images:

Let $\varphi(\xi), \varphi(\psi) \in \Omega$.

Also let $\xi_{0} \in \varphi^{-1}(\varphi(\xi))$ and $\psi_{0} \in \varphi^{-1}(\varphi(\psi))$ be such that $\vDash_{\lambda}\left(\forall \eta\{(\eta \in A)\} ; \eta \in \varphi^{-1}(\varphi(\xi))\right) \rightarrow\left(\xi_{0} \in A\right)$

$\vDash_{\lambda}\left(\forall \eta\{(\eta \in A)\} ; \eta \in \varphi^{-1}(\varphi(\psi))\right) \rightarrow\left(\psi_{0} \in A\right)$

$$
\begin{gathered}
\vDash_{\lambda}(\varphi(\xi) \cdot \varphi(\psi) \in B) \\
\rightarrow\left(\forall \tau\{(\tau \in A)\} ; \tau \in \varphi^{-1}(\varphi(\xi) \cdot \varphi(\psi))\right) \\
\rightarrow\left(\xi_{0} \psi_{0} \in A\right) \\
\rightarrow\left(\xi_{0} \in A\right) \vee\left(\psi_{0} \in A\right) \\
\rightarrow(\varphi(\xi) \in B) \vee(\varphi(\psi) \in B)
\end{gathered}
$$

For pre-images: Let $\xi, \psi \in \Omega$.

$$
\begin{gathered}
F_{\lambda}(\xi \psi \in A) \\
\rightarrow(\varphi(\xi \psi) \in B) \\
\rightarrow(\varphi(\xi) . \varphi(\psi) \in B)
\end{gathered}
$$

Since $\varphi$ is a homomorphism

$$
\rightarrow(\varphi(\xi) \in B) \vee(\varphi(\psi) \in B)
$$

$$
\begin{gathered}
\rightarrow(\xi \in A) \vee(\psi \in A) \\
\vDash_{\lambda}\left(\xi^{-1} \in A\right) \rightarrow\left(\varphi\left(\xi^{-1}\right) \in B\right) \\
\rightarrow\left(\varphi(\xi)^{-1} \in B\right) \\
\rightarrow(\varphi(\xi) \in B) \\
\rightarrow(\xi \in A)
\end{gathered}
$$

\section{Theorem III.4}

Let $\varphi: \Omega \rightarrow \Omega$ be a homomorphism on a finite group $\Omega$. Let $B$ be an implication-based anti-fuzzy normal subgroup of $\varphi(\Omega)$. Then $A=\varphi \circ B$ is also an implication-based anti-fuzzy normal subgroup of $\Omega$.

Proof:

Let $B$ be an implication-based fuzzy normal subgroup of $\varphi(\Omega)$.By Theorem III.3, $A=\varphi \circ B$ is also an implication-based anti-fuzzy subgroup of $\Omega$.

Let $\xi, \psi \in \Omega$

$$
\begin{gathered}
\vDash_{\lambda}(\xi \psi \in A) \\
\rightarrow(\varphi(\xi \psi) \in B) \\
\rightarrow(\varphi(\xi) \varphi(\psi) \in B)
\end{gathered}
$$

Since $\varphi$ is a homomorphism

$$
\rightarrow(\varphi(\psi) \varphi(\xi) \in B)
$$

Since $B$ is an implication-based anti-fuzzy normal subgroup.

$$
\begin{gathered}
\rightarrow(\varphi(\psi \xi) \in B) \\
\rightarrow(\psi \xi \in A)
\end{gathered}
$$

Therefore $A=\varphi \circ B$ is also an implication-based anti-fuzzy normal subgroup of $\Omega$.

\section{PRODUCT OF IMPLICATION-BASED ANTI-FUZZY SUBGROUPS OVER A FINITE GROUP}

\section{Definition IV.1}

Let $\Omega_{1}$ and $\Omega_{2}$ be two finite groups. $\Omega=\Omega_{1} \times \Omega_{2} \$$ be the direct product of $\Omega_{1}$ and $\Omega_{2}$. Then the implication-based anti-fuzzy direct product of the two implication-based anti-fuzzy subgroups $A_{1}$ and $A_{2}$ of $\Omega_{1}$ and $\Omega_{2}$ respectively, is denoted by $A_{1} \times A_{2}$ and is defined as follows

$\vDash_{\lambda}\left(\left(\alpha \in A_{1}\right) \vee\left(\beta \in A_{2}\right)\right) \rightarrow\left((\alpha, \beta) \in A_{1} \times A_{2}\right) \forall \alpha \in \Omega_{1}$ and $\forall \beta \in \Omega_{2}$.

\section{Theorem IV.1}

Let $A$ and $B$ be two implication-based anti-fuzzy subgroups of two finite groups $\Omega_{1}$ and $\Omega_{2}$ respectively. Then $A \times B$ is also an implication-based anti-fuzzy subgroup of $\Omega_{1} \times \Omega_{2}$.

Proof:

Let $\xi=\left(\alpha_{1}, \beta_{1}\right)$ and $\psi=\left(\alpha_{2}, \beta_{2}\right)$ be any two elements of the group $\Omega_{1} \times \Omega_{2}$.

$\vDash_{\lambda}\left(\xi \psi^{-1} \in A \times B\right)$

$\rightarrow\left(\left(\alpha_{1} \alpha_{2}^{-1}, \beta_{1} \beta_{2}{ }^{-1}\right) \in A \times B\right)$

$\rightarrow\left(\alpha_{1} \alpha_{2}{ }^{-1} \in A\right) \vee\left(\beta_{1} \beta_{2}{ }^{-1} \in B\right)$

$\rightarrow\left(\alpha_{1} \in A\right) \vee\left(\alpha_{2}{ }^{-1} \in A\right) \vee\left(\beta_{1} \in B\right) \vee\left(\beta_{2}{ }^{-1} \in B\right)$

$\rightarrow\left(\alpha_{1} \in A\right) \vee\left(\alpha_{2} \in A\right) \vee\left(\beta_{1} \in B\right) \vee\left(\beta_{2} \in B\right)$

$\rightarrow\left(\alpha_{1} \in A\right) \vee\left(\beta_{1} \in B\right) \vee\left(\alpha_{2} \in A\right) \vee\left(\beta_{2} \in B\right)$

$\rightarrow\left(\left(\alpha_{1}, \beta_{1}\right) \in A \times B\right) \vee\left(\left(\alpha_{2}, \beta_{2}\right) \in A \times B\right)$

$\rightarrow(\xi \in A \times B) \vee(\psi \in A \times B)$

By Theorem II.4, $A \times B$ is also an implication-based anti-fuzzy subgroup of $\Omega_{1} \times \Omega_{2}$. 


\section{Theorem IV.2}

Let $A$ and $B$ be two implication-based anti-fuzzy normal subgroups of two finite groups $\Omega_{1}$ and $\Omega_{2}$ respectively. Then $A \times B$ is also an implication-based anti-fuzzy normal subgroup of $\Omega_{1} \times \Omega_{2}$.

Proof:

By Theorem IV.1, $A \times B$ is also an implication-based anti-fuzzy subgroup of $\Omega_{1} \times \Omega_{2}$.

Let $\xi=\left(\alpha_{1}, \beta_{1}\right)$ and $\psi=\left(\alpha_{2}, \beta_{2}\right)$ be any two elements of the group $\Omega_{1} \times \Omega_{2}$.

$\vDash_{\lambda}(\xi \psi \in A \times B) \rightarrow\left(\left(\alpha_{1} \alpha_{2}, \beta_{1} \beta_{2}\right) \in A \times B\right)$

$\rightarrow\left(\alpha_{1} \alpha_{2} \in A\right) \vee\left(\beta_{1} \beta_{2} \in B\right)$

$\rightarrow\left(\alpha_{2} \alpha_{1} \in A\right) \vee\left(\beta_{2} \beta_{1} \in B\right)$

$\rightarrow\left(\left(\alpha_{2} \alpha_{1}, \beta_{2} \beta_{1}\right) \in A \times B\right)$

$\rightarrow(\psi \xi \in A \times B)$

Therefore $A \times B$ is also an implication-based anti-fuzzy subgroup of $\Omega_{1} \times \Omega_{2}$.

\section{Corollary IV.1}

By Theorem II.6 and by Theorem III.3, if $A$ is an implication-based anti-fuzzy normal subgroup of $\Omega$.and $B$ be implication-based anti-fuzzy subgroups of $\Omega$. Then $A \cdot B$ and its pre-image are also an implication-based anti-fuzzy normal subgroups of $\Omega$.

\section{Corollary IV.2}

By Theorem IV.1, Theorem IV.2, Theorem III.3 and Theorem III.4, if $A$ and $B$ are \{implication-based anti-fuzzy normal subgroups of $\Omega$, then $A \times B$ and its pre-image are also an implication-based anti-fuzzy normal subgroups of $\Omega$.

\section{Definition IV.2}

An implication-based anti-fuzzy subgroup A of a finite group $\Omega$ is said to be conjugate to an implication-based anti-fuzzy subgroup $B$ of $\Omega$ if there exists $\psi \in \Omega$ such that $\vDash_{\lambda}(\xi \in A) \rightarrow\left(\psi^{-1} \xi \psi \in B\right)$ for all $\xi \in \Omega$.

\section{Theorem IV.3}

Let an implication-based anti-fuzzy subgroup $A_{1}$ of a finite group $\Omega_{1}$ be conjugate to an implication-based anti-fuzzy subgroup $B_{1}$ of $\Omega_{1}$. Also let an implication-based anti-fuzzy subgroup $A_{2}$ of a finite group $\Omega_{2}$ be conjugate to an implication-based anti-fuzzy subgroup $B_{2}$ of $\Omega_{2}$. Then the implication-based anti-fuzzy subgroup $A_{1} \times A_{2}$ of $\Omega_{1} \times \Omega_{2}$ is conjugate to the implication-based anti-fuzzy subgroup $B_{1} \times B_{2}$ of $\Omega_{1} \times \Omega_{2}$.

Proof:

Let $(\xi, \psi) \in \Omega_{1} \times \Omega_{2}$

$\vDash_{\lambda}\left((\xi, \psi) \in A_{1} \times A_{2}\right)$

$\rightarrow\left(\xi \in A_{1}\right) \vee\left(\psi \in A_{2}\right)$

$\rightarrow\left(\gamma^{-1} \xi \gamma \in B_{1}\right) \vee\left(\delta^{-1} \psi \delta \in B_{2}\right)$

$\rightarrow\left((\gamma, \delta)^{-1} \cdot(\xi, \psi) \cdot(\gamma, \delta) \in B_{1} \times B_{2}\right)$

Therefore theimplication-based anti-fuzzy

subgroupsubgroup $A_{1} \times A_{2}$ of $\Omega_{1} \times \Omega_{2}$ is conjugate to the implication-based anti-fuzzy subgroup $B_{1} \times B_{2}$ of $\Omega_{1} \times \Omega_{2}$.

\section{Theorem IV.4}

Let $A$ and $B$ be two fuzzy subsets of the finite groups $\Omega_{1}$ and $\Omega_{2}$ respectively. Suppose that $\varepsilon_{1}$ and $\varepsilon_{2}$ are the identity elements of $\Omega_{1}$ and $\Omega_{2}$ respectively. If $A \times B$ is an implication-based anti-fuzzy subgroup of $\Omega_{1} \times \Omega_{2}$, then atleast one of the following statement holds.

(i) $\quad \vDash_{\lambda}\left(\varepsilon_{2} \in B\right) \rightarrow(\xi \in A)$ for all $\xi \in \Omega_{1}$

(ii) $\quad \vDash_{\lambda}\left(\varepsilon_{1} \in A\right) \rightarrow(\psi \in B)$ for all $\psi \in \Omega_{2}$

Proof:

$A \times B$ is an implication-based anti-fuzzy subgroup of $\Omega_{1} \times \Omega_{2}$ such that $(i)$ and ( $\left.i i\right)$ conditions does not hold. Then there exists $\xi \in \Omega_{1}$ and all $\psi \in \Omega_{2}$ such that $\vDash_{\lambda}(\xi \in A) \rightarrow\left(\varepsilon_{2} \in B\right)$ for all $\xi \in \Omega_{1}$ $\vDash_{\lambda}(\psi \in B) \rightarrow\left(\varepsilon_{1} \in A\right)$ for all $\psi \in \Omega_{2}$ Let $(\xi, \psi) \in \Omega_{1} \times \Omega_{2}$ $\vDash_{\lambda}((\xi, \psi) \in A \times B) \rightarrow(\xi \in A) \vee(\psi \in B)$

$\rightarrow\left(\varepsilon_{2} \in B\right) \vee\left(\varepsilon_{1} \in A\right)$

$\rightarrow\left(\varepsilon_{1} \in A\right) \vee\left(\varepsilon_{2} \in B\right)$

$\rightarrow\left(\left(\varepsilon_{1}, \varepsilon_{2}\right) \in A \times B\right)$

By Theorem II.5, $A \times B$ is not an implication-based anti-fuzzy subgroup of $\Omega_{1} \times \Omega_{2}$. This is a contradiction to the assumption. Hence either $(i)$ or $(i i)$ should hold.

\section{Theorem IV.5}

Let $A$ and $B$ be two fuzzy subsets of two finite groups $\Omega_{1}$ and $\Omega_{2}$ respectively such that $\vDash_{\lambda}\left(\varepsilon_{2} \in B\right) \rightarrow(\xi \in A)$ for all $\xi \in \Omega_{1}$ where $\varepsilon_{2}$ is the identity element of $\Omega_{2}$. If $A \times B$ is an implication-based anti-fuzzy subgroup of $\Omega_{1} \times \Omega_{2}$, then $A$ is an implication-based anti-fuzzy subgroup of $\Omega_{1}$.

Proof:

Let $A \times B$ be an implication-based anti-fuzzy subgroup of

$\Omega_{1} \times \Omega_{2}$. Let $\xi, \psi \in \Omega_{1}$.

$\Rightarrow\left(\xi, \varepsilon_{2}\right),\left(\psi, \varepsilon_{2}\right) \in \Omega_{1} \times \Omega_{2}$

$\vDash_{\lambda}(\xi \psi \in A)$

$\rightarrow(\xi \psi \in A) \vee\left(\varepsilon_{2} \varepsilon_{2} \in B\right)$

$\rightarrow\left(\left(\xi \psi, \varepsilon_{2} \varepsilon_{2}\right) \in A \times B\right)$

$\rightarrow\left(\left(\xi, \varepsilon_{2}\right) \cdot\left(\psi, \varepsilon_{2}\right) \in A \times B\right)$

$\rightarrow\left(\left(\xi, \varepsilon_{2}\right) \in A \times B\right) \vee\left(\left(\psi, \varepsilon_{2}\right) \in A \times B\right)$

$\rightarrow(\xi \in A) \vee\left(\varepsilon_{2} \in B\right) \vee(\psi \in A) \vee\left(\varepsilon_{2} \in B\right)$

$\rightarrow(\xi \in A) \vee(\psi \in A)$

$\vDash_{\lambda}\left(\xi^{-1} \in A\right) \rightarrow\left(\xi^{-1} \in A\right) \vee\left(\varepsilon_{2}^{-1} \in B\right)$

$\rightarrow\left(\left(\xi^{-1}, \varepsilon_{2}^{-1}\right) \in A \times B\right)$

$\rightarrow\left(\left(\xi, \varepsilon_{2}\right)^{-1} \in A \times B\right)$

$\rightarrow\left(\left(\xi, \varepsilon_{2}\right) \in A \times B\right)$

$\rightarrow(\xi \in A) \vee\left(\varepsilon_{2} \in B\right)$

$\rightarrow(\xi \in A)$

Therefore $A$ is an implication-based anti-fuzzy subgroup of $\Omega_{1}$.

\section{Corollary IV.3}

Let $A$ and $B$ be two fuzzy subsets of two finite groups $\Omega_{1}$ and $\Omega_{2}$ respectively such that $\vDash_{\lambda}\left(\varepsilon_{1} \in A\right) \rightarrow(\xi \in B)$ for all $\xi \in \Omega_{2}$ where $\varepsilon_{1}$ is the identity element of $\Omega_{1}$. If $A \times B$ is an implication-based anti-fuzzy subgroup of $\Omega_{1} \times \Omega_{2}$, then $B$ is an implication-based anti-fuzzy subgroup of $\Omega_{2}$.

\section{Corollary IV.4}

By Theorem IV.4, Theorem IV.5 and Corollary IV.3 we have, let $A$ and $B$ be fuzzy subsets of the finite groups $\Omega_{1}$ and $\Omega_{2}$ respectively. If $A \times B$ is an implication-based anti-fuzzy subgroup of $\Omega_{1} \times \Omega_{2}$, then either $A$ is an implication-based anti-fuzzy subgroup of $\Omega_{1}$, or $B$ is an implication-based anti-fuzzy subgroup of $\Omega_{2}$. 


\section{CONCLUSION}

In this paper, the notion of implication-based anti-fuzzydirect product of implication-based anti-fuzzy subgroup of a finite group is introduced. It is proved that union of two implication-based anti-fuzzy subgroups of a finite group is also an implication-based anti-fuzzy subgroup. Further, the homomorphic pre-image of implication-based anti-fuzzy normal subgroup of a finite group is also an implication-based anti-fuzzy normal subgroup. Certain algebraic properties of implication-based anti-fuzzy direct product of implication-based anti-fuzzy subgroups of a finite group are also proved.

\section{REFERENCES}

1. Biswas R, "Fuzzy Subgroups and Anti-Fuzzy Subgroup", Fuzzy Sets and Systems, Vol 35, 1990, pp $121-124$.

2. B. O. Onasanya, "Review on Some Anti-Fuzzy Properties of Some Fuzzy Subgroup", Annals of Fuzzy Mathematics and Informatics, Vol. 11, No. 6, 2016, pp $899-904$.

3. D. S. Malik, John. N. Mordeson and P. S. Nair, "Fuzzy Normal Subgroups in Fuzzy Subgroups ", J. Korean Math. Soc, Vol. 29, 1992, pp $1-8$.

4. K. A. Dib and A . A. Hassan, "The Fuzzy Normal Subgroup ", Fuzzy Sets and Systems, Vol. 98, 1998, pp 393-402.

5. Kyung Ho Kim and Young Bae Jun, "Anti Fuzzy R - Subgroups of Near Rings ", Scientiae Mathematicae, Vol. 2, No. 2, 1999, pp 147 - 153.

6. Mingsheng Ying, " A New Approach For Fuzzy Topology (I)", Fuzzy Sets and Systems, Vol 39, 1997, pp 303 - 321.

7. N.P. Mukherjee and Prabir Bhattacharya, "Fuzzy Normal Subgroups and Fuzzy Cosets ", Information Sciences, Vol. 34, 1984, pp 225 - 239

8. P. K. Sharma, "" $(\alpha, \beta)$ - Anti Fuzzy Subgroups ",International Journal of Fuzzy Mathematics and Systems, Vol. 3, No. 1, 2013, pp 61 - 74.

9. P. M. Sithar Selvam, T. Priya, K. T. Nagalakshmi and T. Ramachandran, "On Some Properties of Anti-Q-Fuzzy Normal Subgroups ", Gen. Math. Notes, Vol. 22, No. 1, 2014, pp $1-10$.

10. Rosenfeld, A., "Fuzzy Groups", Journal of Mathematical Analysis and applications, Vol 35, 1971, pp 512-517.

11. Selvarathi, M \& Spinneli, M. A. J., "Implication-Based Fuzzy Normal Subgroup of a Finite Group", International Journal of Applied Engineering Research, Vol. 10, No. 80, 2015, pp 5-8.

12. Selvarathi, M \& Spinneli, M. A. J.," Implication-Based Anti-Fuzzy Normal Subgroup of a Finite Group ", International Journal of Pure and Applied Mathematics, Vol. 119, No. 9, 2018, pp 1 - 9.

13. Umer Shuaib and Muhammad Shaheryar, "On Some Properties of o-Anti Fuzzy Subgroups", International Journal of Mathematics and Computer Science, Vol. 14, No. 1, 2019, pp 215 - 230.

14. Xuehai Yuan, Cheng Zhang \& Yonghong Ren, "Generalized fuzzy groups and many-valued implications",Fuzzy Sets and System, Vol 138, 2003, pp 205-211.

15. Yuming Feng and Bingxue Yao, "On $(\lambda, \mu)$ - anti-fuzzy subgroups ", Journal of Inequalities and Applications, Vol. 78,2012, pp 1 - 5.

16. Zadeh L. A., "Fuzzy Sets", Information Control, Vol 8, 1965, pp 338 353.

\section{AUTHORS PROFILE}

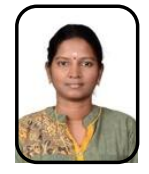

Dr. M. Selvarathi received the Doctor of Philosophy in Mathematics from Karunya Institute of Technology and Sciences (Deemed to be University), Coimbatore, India. She is currently working as an Assistant Professor of Mathematics in Karunya Institute of Technology and Sciences, India. She is involved in an elaborate research in the field of fuzzy automata. Her research findings are published in reputed international journals. 